\title{
Detection of Y-Chromosome microdeletions in Egyptian infertile males
}

\section{Azza S. Ahmed ${ }^{1}$ Mohamed A. El-Dessouky² Abdelgawad A. Fahmi ${ }^{2}$ Fatma A. EIRefaey $^{3}$ and Yasser H. EINahass ${ }^{3}$}

${ }^{1,2}$ Chesmistry Department, Faculty of Science, Cairo University, Egypt

${ }^{3}$ Clinical Pathology Department, National Cancer Institute, Cairo University, Egypt.

\section{Abstract}

The major genetic causes in male infertility are chromosomal abnormalities and $\mathrm{Y}$ chromosomal microdeletions (YCMs). YCMs occur in approximately 15\% of azoospermic patients and $10 \%$ of severe oligospermic patients. These microdeletions lead to spermatogenic failure.

This study aims to report the incidence of Azoospermia factor (AZF) microdeletions in Egyptian infertile males with severe oligoospermia \& non obstructive azoospermia (NOA) using multiplex PCR.

One hundred-fifty infertile males were included. Semen analysis, hormonal assay, karyotyping, testicular sperm extraction and testicular biopsy were performed.Y chromosome microdeletions were detected by using multiplex polymerase chain reaction (PCR).

Among 150 infertile males; Considering Y chromosome; in severe oligospermic infertile males 3/36 (8.3\%) had Y chromosome microdeletions in AZF subregions where; 1/3(33.3\%) showed deletions in AZF-c and 2/3(66.7\%) showed deletions in both AZF-b+c. However; no deletions were detected in AZF-a region in this group. In NOA group, 21/114(18.4\%) had Y chromsome microdeletions in AZF subregions where; 1/21 (4.8\%) showed deletions in AZF$\mathrm{b}$ region, $2 / 21(9.5 \%)$ showed deletion in both of AZF-a+b+c regions, 8/21 (38\%) showed deletions in AZF-c region only and 10/21 (47.6\%) showed deletions in both AZF-b+c regions.

Conclusion: The frequency of $\mathrm{Y}$ chromosome microdeletions in our studied patients was similar to many ethnic reports. Detection of AZF microdeletions is necessary for proper genetic diagnosis in infertile males. AZFc can help informed decisions regarding positive testicular sperm extraction outcome.

Keywords: Male infertility, Y chromosome microdeletions, AZF, NOA, TESE

\section{Introduction:}

Infertility is a major problem affects approximately $15 \%$ of couples around the world [1]. The failure of conception after 12 months of unprotected intercourse is known as infertility [2]. Male infertility divided into primary infertility and secondary infertility [3]. Major causes of infertility are idiopathic infertility, testicular failure, obstruction, cryptorchidism, low semen volume, sperm agglutination, varicocele, erectile or ejaculatory dysfunction, abnormal viscosity, endocrine disorder, high density of sperm, environmental causes and genetic abnormalities [4]. Genetic factors play well-recognized roles in male infertility, and genetic alterations of the $\mathrm{Y}$ chromosome are especially important [5].

Chromosomal anomalies and Y chromosomal microdeletions (YCMs) are the main genetic 
factors in male infertility. YCMs occur in approximately $15 \%$ of azoospermic patients and $10 \%$ of severe oligospermic patients, and are frequently found at the azoospermia factor (AZF) locus in the q11.23 band. The AZF locus was subdivided by molecular analyses into three (probably Four) sub regions: $\mathrm{AZFa}, \mathrm{AZFb}$ and $\mathrm{AZFc}$, with a fourth probable AZFd region [6]. AZFc region is haboring Candidate genes that include a cluster of four genes termed the Deleted in Azoospermia (DAZ) cluster and expressed only in germ cells [7].

As for the $\mathrm{AZFb}$ region, it includes a family of genes known as the RNA-binding motif (RBMY) family whose expression is also restricted to the testis $[8,9,10,11]$. Microdeletions in these regions lead to different degrees of spermatogenetic failure; although, the exact genotype phenotype relationship of microdeletions and AZF gene function and infertility in the AZF locus have not been fully discovered [12].

Genes that control sex differentiation are carried on Sex-determining region Y (SRY) present on Y chromosome short arm (Yp). Other genes affecting spermatogenesis have been found on another region on (Yq), known as AZF which has been correlated to male infertility. AZF deletion were first observed by in azoospermic infertile males [11]. AZF microdeletions reveals the possibility of spermatogenesis related genes spontenous loss $[12,13]$

The purpose of this work was to report the incidence of AZF microdeletions in Egyptian infertile males with severe oligoospermia and non obstructive azoospermia (NOA) using multiplex PCR.

\section{Patients and Methods}

Between May 2016 and October 2018, 150 infertile males who presented to the Andrology Department, Kasr El-Einy, Cairo University, were included. Their median age was 36.0 years (19-54). The required documents are submitted according to the committee guidelines \& have been received \&showed by Central Directorate of Research \& Health Development and the committee decision is Approval Com. No/Dec. No: 5-2019/4

All-purpose clinical records and blood samples were obtained in addition to complete semen analysis according to WHO 2010 criteria [14]. Based on semen analysis, participants were classified into two groups: Non obstructive azoospermia (zero sperm count) and severe Oligozoospermia ( $<5$ million sperm count $/ \mu l$ ). Testicular biopsies were collected and divided into four different groups; Mixed sertoli pattern , Primary spermatocyte arrest, Sertoli cell only syndrome and Hypospermatogenesis [15]. Hormonal profile for each patient was evaluated including: follicle-stimulating hormone (FSH), luteinizing hormone ( $\mathrm{LH}$ ), and testosterone levels and performed by enzyme immunoassay sandwich method with a final fluorescent detection (ELFA)[11]. Karyotyping was performed by G-banding techniques according to standard methods as described by [16]. Patients with normal (46, XY) and abnormal Klienfilter's syndrome (47, XXY) karyotypes were included. Testicular sperm extraction (TESE) was performed for some patients with results recorded as positive or negative trial. 


\subsection{Specimen:}

\section{A- Blood}

a. $3 \mathrm{ml}$ whole blood on ethylene diamine tetra acetic acid (EDTA) tube for DNA extraction and multiplex PCR screening of Y-chromosome microdeletions;

b. $6 \mathrm{ml}$ whole blood divided into two lithium heparin tubes $3 \mathrm{ml}$ per each one (duplicate) for karyotyping to detect any chromosomal aberration.

c. $3 \mathrm{ml}$ whole blood on plain tube was left for 15 minutes at water bath then centrifuged and serum was withdrawn for hormonal assay (FSH, LH, and T. TT).

\section{B-Semen:}

For all 150 Seminal analyses were done to classify the infertility case into two main groups; whether Non-obstructive Azoospermia (NOA) or severe oligospermia.

Semen samples were collected by masturbation in sterile cups following 3 days with no sexual intercourse. Semen samples were let to liquefy for 20 minutes, and then volume, concentration, total spermatic count, motility, and abnormalities were evaluated.

\section{C-Testicular biopsy}

Some patient underwent testicular pathology, was done for NOA infertile males to reflect spermatogenesis state and determine to which category it belongs whether sertoli cell only syndrome (SCOS), Maturation arrest, mixed sertoli or hypospermatogeneis Patients' samples were sent to histopathology lab and results were recorded.

\subsection{DNA extraction:}

Genomic DNA was extracted using Gentra Puregene Blood Kit (Qiagen, Germany). $900 \mu \mathrm{l}$ RBCs lysis solution was added to $300 \mu l$ of whole blood followed by mixing by inversion for 10 times.

\subsection{Multiplex PCR for sequence tagged sites (STSs):}

Polymerase chain reaction (PCR) amplification using 25 sequences tagged sites (STSs) within the AZF region of Yq11 were used in five multiplex PCR sets for AZF microdeletions detection of the $\mathrm{Y}$ chromosome. Each multiplex set contained five pairs (forward and reverse) of different designed primer (Bio Basic Inc., Canada).in order to cover euchromatic region of Yq11 (AZFa, AZFb and AZFc regions) where microdeletions might take place. Multiplex polymerase chain reactions were prepared in five different mixes; A, B, C, D and E

Briefly, each PCR mixes contained 500 ng genomic DNA added to hot start master mix MyTaq $^{\text {TM }}$ HS Red Mix (2x, Bioline, London, UK)containing (10x Buffer, dNTPs\& hot start enzyme) in addition to $0.8 \mu M$ of forward and reverse primers and adjusted with distilled water into a total reaction volume of $25 \mu \mathrm{l}$. 
Amplification was performed in a T-Personal thermal cycler (Biometra Göettingen, Germany).The amplification protocol was as follows: initial denaturation at $95^{\circ} \mathrm{C}$ for $10 \mathrm{~min}$, a consequent series of 45 cycles at $94^{\circ} \mathrm{C}$ for $45 \mathrm{sec}$ (denaturation), $60^{\circ} \mathrm{C}$ for 1 min (annealing), and $72^{\circ} \mathrm{C}$ for $2 \mathrm{~min}$ (extension). A final extension was carried out at $72^{\circ} \mathrm{C}$ for $7 \mathrm{~min}$. A STS for SRY (sex-determining region on the Y-chromosome SRY gene) sY14 was used as a control primer to distinguish a negative result from a technical failure. Female genomic DNA which controls for specificity and contamination was used as a negative control. No template control (NTC) was used to check for contamination in every PCR reaction.

Table 1: Multiplex sets containing 25 sequences tagged sites (STSs) used for detecting Y microdeletions

\begin{tabular}{|c|c|c|c|c|}
\hline $\begin{array}{l}\text { Multipl } \\
\text { ex set }\end{array}$ & STSs & Left primer & Right primer & $\begin{array}{l}\text { Fragm } \\
\text { ent size } \\
\text { in (bp) }\end{array}$ \\
\hline $\begin{array}{l}\text { Multipl } \\
\text { ex PCR } \\
\quad \text { I }\end{array}$ & $\begin{array}{c}\text { sY27 } \\
2 \\
\text { sY15 } \\
2 \\
\text { sY13 } \\
2 \\
\text { sY84 } \\
\text { sY14 }\end{array}$ & $\begin{array}{c}\text { GGTGAGTCAAATTAGTCAAT } \\
\text { GTCC } \\
\text { AAGACAGTCTGCCATGTTTA } \\
\text { GAGAGTCATAATGCCGACG } \\
\mathbf{T} \\
\text { AGAAGGGTCTGAAAGCAGG } \\
\mathbf{T} \\
\text { GAATATTCCCGCTCTCCGGA }\end{array}$ & $\begin{array}{c}\text { CCTTACCACAGGACAGAGG } \\
\text { G } \\
\text { ACAGGAGGGTACTTAGCAG } \\
\text { T } \\
\text { TGGTCTCAGGAAGTTTTTG } \\
\text { C } \\
\text { GCCTACTACCTGGAGGCTT } \\
\text { C } \\
\text { GCTGGTGCTCCATTCTTGA } \\
\text { G }\end{array}$ & $\begin{array}{c}93 \\
125 \\
143 \\
326 \\
472\end{array}$ \\
\hline $\begin{array}{l}\text { Multipl } \\
\text { ex PCR } \\
\text { II }\end{array}$ & $\begin{array}{c}\text { sY26 } \\
9 \\
\text { sY13 } \\
9 \\
\text { sY15 } \\
3 \\
\text { sY13 } \\
8 \\
\text { sY15 } \\
5\end{array}$ & $\begin{array}{c}\text { CTCTGGGACAAGTGTTCCTT } \\
\text { G } \\
\text { TTCAGAGGAATCATGTGGG } \\
\text { T } \\
\text { GCATCCTCATTTTATGTCCA } \\
\text { CACATGAAGCACTGGAACT } \\
\text { G } \\
\text { ATTTTGCCTTGCATTGCTAG }\end{array}$ & $\begin{array}{c}\text { CATTGGCATGAATGTGTAT } \\
\text { TCA } \\
\text { AATGTTTCATCACCATTAT } \\
\text { CCC } \\
\text { CAACCCAAAAGCACTGAGT } \\
\text { A } \\
\text { AGGGCCTGAGTCTCCAGG } \\
\text { TTTTTAAGCCTGTGACCTG } \\
\text { G }\end{array}$ & $\begin{array}{c}94 \\
120 \\
139 \\
170 \\
349\end{array}$ \\
\hline $\begin{array}{l}\text { Multipl } \\
\text { ex PCR } \\
\text { III }\end{array}$ & $\begin{array}{c}\text { sY25 } \\
5 \\
\text { sY14 } \\
4 \\
\text { sY16 } \\
0 \\
\text { sY14 } \\
3 \\
\text { sY25 } \\
4\end{array}$ & $\begin{array}{c}\text { GTTACAGGATTCGGCGTGA } \\
\text { T } \\
\text { TCATCTGCCACCATCAACAT } \\
\text { TACGGGTCTCGAATGGAAT } \\
\text { A } \\
\text { GCAGGATGAGAAGCAGGTA } \\
\text { G } \\
\text { GGGTGTTACCAGAAGGCAA } \\
\text { A }\end{array}$ & $\begin{array}{c}\text { CTCGTCATGTGCAGCCAC } \\
\text { ACGTGTTTCTACACCTGCC } \\
\text { C } \\
\text { TCATTGCATTCCTTTCCATT } \\
\text { CCGTGTGCTGGAGACTAAT } \\
\text { C } \\
\text { GAACCGTATCTACCAAAGC } \\
\text { AGC }\end{array}$ & $\begin{array}{l}126 \\
143 \\
236 \\
311 \\
350\end{array}$ \\
\hline Multipl & $\begin{array}{l}\text { sY27 } \\
3 \\
\text { sY24 }\end{array}$ & $\begin{array}{c}\text { GGTCTTTAAAAGGTGAGTCA } \\
\text { AATT } \\
\text { GTTTCTTCATAAGCAACCAA }\end{array}$ & $\begin{array}{c}\text { AGACAGAGGGAACTTCAAG } \\
\text { ACC } \\
\text { CAGATTATGCCACTGCCCT }\end{array}$ & $\begin{array}{c}93 \\
118 \\
400\end{array}$ \\
\hline
\end{tabular}




\begin{tabular}{|c|c|c|c|c|}
\hline $\begin{array}{c}\text { ex PCR } \\
\text { IV }\end{array}$ & $\begin{array}{l}3 \\
\text { SPG } \\
\text { Y } \\
\text { SY16 } \\
4 \\
\text { RB } \\
\text { M1 }\end{array}$ & $\begin{array}{c}\text { ATTG } \\
\text { TTTCACATACAGCCATTAAG } \\
\text { TTTAGC } \\
\text { AATGTGCCCACACAGAGTTC } \\
\text { ATGCACTTCAGAGATACGG }\end{array}$ & $\begin{array}{c}\text { T } \\
\text { CAATTTTGATAGTCTGAAC } \\
\text { ACAAGC } \\
\text { TGGAAGACCAGGATTTCAT } \\
\text { G } \\
\text { CCTCTCTCCACAAAACCAA } \\
\text { CA }\end{array}$ & $\begin{array}{l}590 \\
800\end{array}$ \\
\hline $\begin{array}{c}\text { Multipl } \\
\text { ex PCR } \\
\text { V }\end{array}$ & $\begin{array}{r}\mathrm{s} Y 16 \\
6 \\
\mathrm{~s} Y 15 \\
0 \\
\mathrm{~s} Y 15 \\
8 \\
\mathrm{~s} Y 11 \\
7 \\
\mathrm{~s} Y 27 \\
7\end{array}$ & $\begin{array}{c}\text { GAACTCCAATCATTCCCTGA } \\
\text { GGGAGAGTCACATCACTTG } \\
\text { G } \\
\text { CTCAGAAGTCCTCCTAATAG } \\
\text { TTCC } \\
\text { GTTGGTTCCATGCTCCATAC } \\
\text { GGGTTTTGCCTGCATACGTA } \\
\text { ATTA }\end{array}$ & $\begin{array}{c}\text { TTGGCTCTACTTTTCCCCTT } \\
\text { TTGAATTATCTGCCTGAGT } \\
\text { GC } \\
\text { ACAGTGGTTTGTAGCGGGT } \\
\text { A } \\
\text { CAGGGAGAGAGCCTTTTAC } \\
\text { C } \\
\text { CCTAAAAGCAATTCTAAAC } \\
\text { CTCCAG }\end{array}$ & $\begin{array}{l}115 \\
158 \\
231 \\
262 \\
310\end{array}$ \\
\hline
\end{tabular}

\subsection{Detection of amplified product using gel electrophoresis:}

A 2\% agarose gel (GenAgarose L.E. Genaxxon bioscience GmbH Söflinger, Germany) was prepared in 1x TAE buffer (Bio Basic Inc., Canada) mixed with $4 \mu$ l ethidium bromide. 12.5 $\mu \mathrm{l}$ of the final PCR product were electrophoresed with Gene DireX ${ }^{\circledR} 50$ bp ladder RTU (ready to use) by GeneDirex, Co. on a MultiSUB Horizontal Gel Systems andnanoPAC-300 (Cleaver Scientific LTD, United Kingdom) connected to supply voltage power which was adjusted at $160 \mathrm{~V}$ for $25 \mathrm{~min}$ at room temperature. The gel was visualized under ultraviolet light of UV trans-illuminator (Spectroline, New York).

\section{Results:}

One hundred fifty infertile males were included in this study with a median age of 36.0 years (19-56). Based on semen analysis, patients were divided into two groups; nonobstructive azoospermia 114/150 (76\%) and severe oligozoospermia 36/150 (24\%). TESE was performed for 102/150(68\%) patients, 25/150 had a successful TESE (16.7\%). Karyotyping was performed for $26 / 36(72.2 \%)$ of oligospermic and 74/114 (65\%) of NOA patients, results showed both normal karyotype (46, XY) and abnormal karyotype (47, XXY) where Klinefelter's syndrome. 
Table 2: Patients characteristics

\begin{tabular}{|c|c|}
\hline Parameters & Percentages \\
\hline $\begin{array}{r}\text { Age (years) }(\text { Mean } \pm \text { SD) } \\
\text { NOA } \\
\text { Severe oligospermia }\end{array}$ & $\begin{array}{l}35.17 \pm 7.85 \\
35.72 \pm 6.48\end{array}$ \\
\hline \multicolumn{2}{|c|}{ Semen analysis } \\
\hline \multirow{2}{*}{$\begin{array}{r}\text { NOA } \\
\text { Severe Oligozoospermia } \\
\end{array}$} & $114(76 \%)$ \\
\hline & $36(24 \%)$ \\
\hline \multicolumn{2}{|c|}{ FSH } \\
\hline $\begin{array}{r}\text { NOA } \\
\text { Severe oligospermia }\end{array}$ & $\begin{array}{l}14.70 \pm 9.45 \\
14.32 \pm 7.93\end{array}$ \\
\hline \multicolumn{2}{|c|}{$\mathbf{L H}$} \\
\hline \multirow{2}{*}{$\begin{array}{r}\text { NOA } \\
\text { Severe oligospermia }\end{array}$} & $9 \pm 5.27$ \\
\hline & $7.75 \pm 3.85$ \\
\hline \multicolumn{2}{|c|}{ Testosterone } \\
\hline \multirow{2}{*}{$\begin{array}{r}\text { NOA } \\
\text { Severe oligospermia }\end{array}$} & $3.47 \pm 2.45$ \\
\hline & $3.16 \pm 2.23$ \\
\hline \multicolumn{2}{|c|}{ Testicular sperm extraction } \\
\hline \multirow{2}{*}{$\begin{array}{r}\text { Positive } \\
\text { Negative }\end{array}$} & $25(16.7 \%)$ \\
\hline & $77(75.3 \%)$ \\
\hline \multicolumn{2}{|c|}{ Karyotype } \\
\hline \multirow{2}{*}{$\begin{array}{r}\text { Normal } \\
\text { Abnormal }\end{array}$} & $83(83 \%)$ \\
\hline & $17(17 \%)$ \\
\hline
\end{tabular}

\section{Y chromosome microdeletions:}

Y chromosome microdeletions were detected in twenty-four patients 24/150 (16\%) had microdeletions; $3 / 150(2 \%)$ had severe oligospermia while $21 / 150(14 \%)$ had NOA. A total of 15 deleted STS were detected; we used 25 STSs for AZF microdeletions detection, $7 / 25(28 \%)$ deleted STSs of oligospermic group were detected. In NOA group, a total of 15/25 (60\%) deleted STS were detected as shown in details in table (3) \& (4)

Table 3: distribution of different STS microdeletions in 21/114 (18.4\%) of NOA.

\begin{tabular}{|l|l|l|l|}
\hline $\begin{array}{l}\text { No. of cases } \\
21 / 114\end{array}$ & AZF-a & AZF-b & AZF-c \\
\hline 1 & & & Sy277, Sy158 \\
\hline 2 & & Sy143 & Sy254, Sy158, Sy166 \\
\hline 3 & & & Sy243, Sy158 \\
\hline 4 & & & Sy152, Sy243, Sy273 \\
\hline 5 & & Sy117 & \\
\hline
\end{tabular}




\begin{tabular}{|l|l|l|l|}
\hline 6 & & & Sy166 \\
\hline 7 & Sy84 & Sy117 & Sy277, Sy243 \\
\hline 8 & & Sy11, Sy132 & Sy277, Sy243, Sy158, Sy272, Sy273, Sy152 \\
\hline 9 & & Sy117 & Sy277, Sy243, Sy144, Sy254 \\
\hline 10 & & Sy117 & Sy277 \\
\hline 11 & & & Sy273 \\
\hline 12 & & & Sy273 \\
\hline 13 & & & Sy273 \\
\hline 14 & & Sy117 & Sy254, Sy158, Sy273, Sy160 \\
\hline 15 & Sy84 & Sy117, Sy132 & Sy254, Sy277, Sy243, Sy158, Sy272 \\
\hline 16 & & Sy117, Sy132 & Sy277, Sy243, Sy272 \\
\hline 17 & & Sy117, Sy132 & Sy255, Sy277 \\
\hline 18 & & Sy117 & Sy277, Sy243, Sy158, Sy272 \\
\hline 19 & & Sy117 & Sy277 \\
\hline 20 & & & Sy277 \\
\hline 21 & & Sy117 & Sy277, Sy243, Sy273 \\
\hline
\end{tabular}

Table 4: The distribution of AZF microdeletions in subregions a, b and $c$ in both groups.

\begin{tabular}{|c|c|c|c|c|}
\hline \multirow{2}{*}{ Groups } & \multicolumn{4}{|c|}{ Azoospermia factor-AZF } \\
\cline { 2 - 5 } & $\begin{array}{c}\text { AZF- } \\
\text { AZF }\end{array}$ & $\begin{array}{c}\text { AZF- } \\
\text { AZF- }\end{array}$ & $\begin{array}{c}\text { AZF- } \\
\text { b+c }\end{array}$ & \begin{tabular}{c} 
a+b+c \\
\hline Severe
\end{tabular} \\
\hline Oligoospermia & & $1 / 3$ & $2 / 3$ & \\
\hline NOA & $1 / 21$ & $8 / 21$ & $10 / 21$ & $2 / 21$ \\
$(4.8 \%)$ & $(38 \%)$ & $(47.6 \%)$ & $(9.5 \%)$ \\
\hline
\end{tabular}




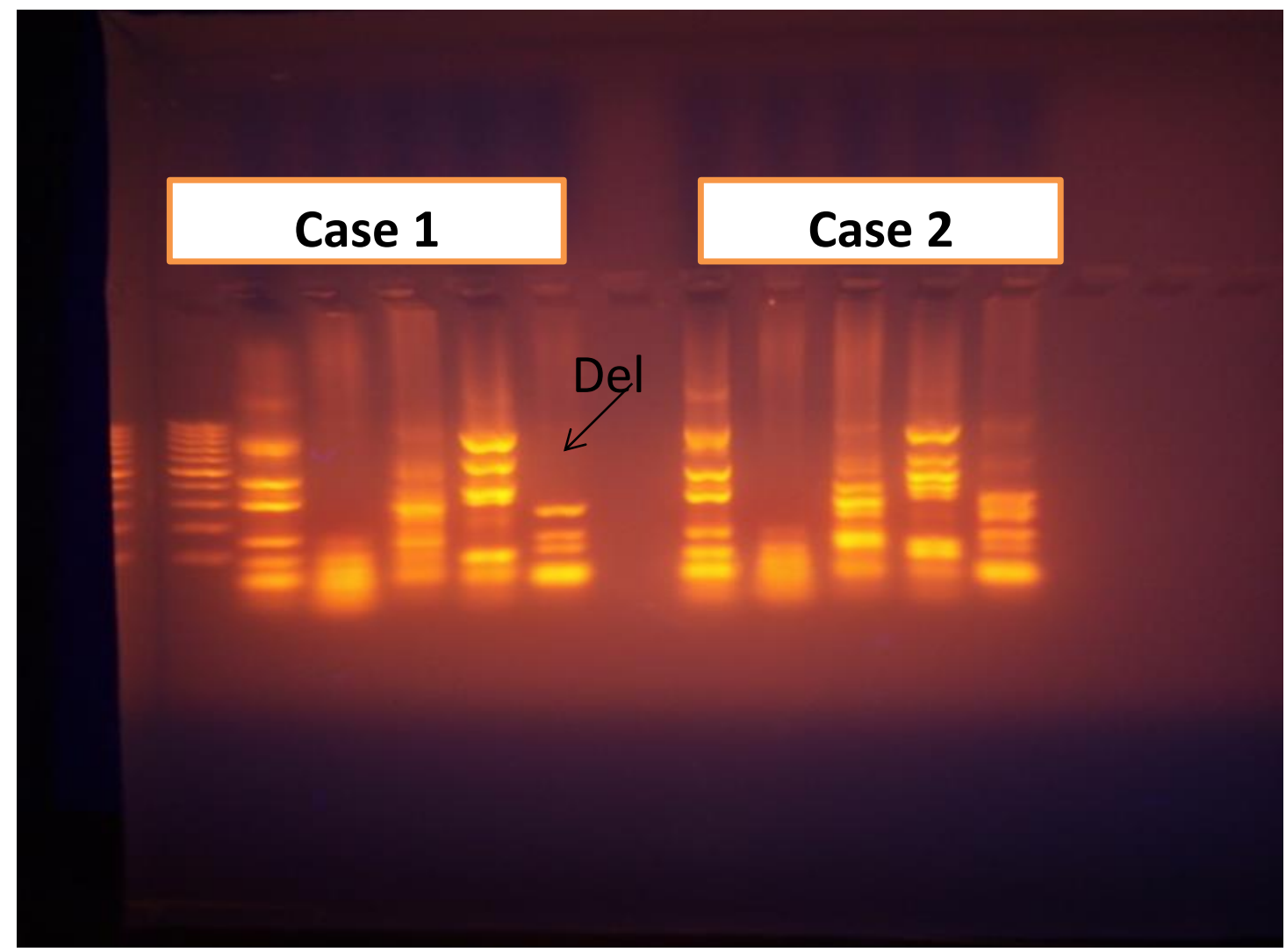

Figure 1: Gel electrophoresis for included infertile male with Y-Chromsome microdeletions in Case 1 showed deletion in Sy277 and sY117 while Case 2; showed normal infertile male.

Table 5: The distribution of $Y$ microdeletions correlated to Karyotyping and TESE.

\begin{tabular}{|c|l|l|l|l|l|l|}
\hline \multirow{2}{*}{ Groups } & \multicolumn{2}{|c|}{ Karyotyping } & \multicolumn{2}{c|}{ TESE } & \multicolumn{2}{c|}{$\begin{array}{l}\text { Y chromosome } \\
\text { microdeletions }\end{array}$} \\
\cline { 2 - 7 } & $\begin{array}{c}\text { Normal } \\
(46, \text { XY) }\end{array}$ & $\begin{array}{l}\text { Abnormal } \\
\text { Klienfilter' } \\
\text { syndrome } \\
(47, \text { XXY) }\end{array}$ & Negative & Positive & No deletion & Microdeletion \\
\hline $\begin{array}{c}\text { Severe } \\
\text { Oligoospermia }\end{array}$ & $\begin{array}{l}20 / 26 \\
(77 \%)\end{array}$ & $\begin{array}{l}6 / 26 \\
(23 \%)\end{array}$ & $\begin{array}{l}5 / 8 \\
(62.5 \%)\end{array}$ & $\begin{array}{l}3 / 8 \\
(37.5 \%)\end{array}$ & $\begin{array}{l}33 / 36 \\
(91.7 \%)\end{array}$ & $\begin{array}{l}3 / 36 \\
(8.3 \%)\end{array}$ \\
\hline NOA & $\begin{array}{l}63 / 74 \\
(85 \%)\end{array}$ & $\begin{array}{l}11 / 74 \\
(15 \%)\end{array}$ & $\begin{array}{l}72 / 94 \\
(76.6 \%)\end{array}$ & $\begin{array}{l}22 / 94 \\
(23.4 \%)\end{array}$ & $\begin{array}{l}93 / 114 \\
(81.6 \%)\end{array}$ & $\begin{array}{l}21 / 114 \\
(18.4 \%)\end{array}$ \\
\hline $\begin{array}{c}\text { N (\%) within Y } \\
\text { micro deletion }\end{array}$ & $\begin{array}{c}9 / 12 \\
(75 \%)\end{array}$ & $\begin{array}{c}3 / 12 \\
(25 \%)\end{array}$ & $\begin{array}{c}17 / 19 \\
(89.5 \%)\end{array}$ & $\begin{array}{l}2 / 19(10.5 \\
\%)\end{array}$ & & \\
\hline
\end{tabular}


Table 6: Distribution of Y microdeletions correlated to histopathological patterns

\begin{tabular}{|c|c|c|c|c|c|}
\hline & & \multicolumn{4}{|c|}{ Histological patterns of testicular biopsies } \\
\hline & & $\begin{array}{c}\text { Sertoli } \\
\text { cell } \\
\text { only } \\
\text { syndrome }\end{array}$ & $\begin{array}{c}\text { Primary } \\
\text { spermatocyte } \\
\text { arrest }\end{array}$ & $\begin{array}{c}\text { Mixed } \\
\text { sertoli } \\
\text { pattern }\end{array}$ & Hypospermatogenesis \\
\hline \multirow{2}{*}{$\begin{array}{l}\text { Deletions in } \\
\text { Y- } \\
\text { chromosome }\end{array}$} & Number & 7 & 7 & 4 & 3 \\
\hline & $\begin{array}{c}\mathrm{N}(\%) \\
\text { within Y } \\
\text { micro } \\
\text { deletion }\end{array}$ & $\begin{array}{c}1 / 6 \\
(16.7 \%)\end{array}$ & $\begin{array}{c}2 / 6 \\
(33.3 \%)\end{array}$ & $\begin{array}{c}1 / 6 \\
(16.7 \%)\end{array}$ & $\begin{array}{c}2 / 6 \\
(33.3 \%)\end{array}$ \\
\hline
\end{tabular}

\section{Correlation of $Y$ chromosome microdeletions with Hormonal assesment:}

In oligoospermic group, FSH with $10.60 \pm 0.95 \mathrm{mIu} / \mathrm{ml}$, LH with $6.40 \pm 1.98) \mathrm{mIu} / \mathrm{ml}$ and total testosterone with $6.05 \pm 3.39 \mathrm{mIu} / \mathrm{ml}$ while in NOA group FSH with $11.85 \pm 6.78 \mathrm{mIu} / \mathrm{ml}$, LH with $7.187 \pm 3.75 \mathrm{mIu} / \mathrm{ml}$ and total testosterone with $4.29 \pm 2.76 \mathrm{mIu} / \mathrm{ml}$.

Statistical analysis showed there was no significant difference in hormonal level between both groups.

\section{Discussion}

Nowadays Y-chromosome microdeletions are considered a potential genetic cause of male infertility [3]. In our study, 24/150 (16\%) patients had microdeletions. Previous studies reported different frequencies in Y microdeletions; 24.2\% [17], 50\% [18], 12\% [19], 5\% [20], $6 \%[21], 5.2 \%[22],[5.4 \%[23], 5 \%$ [24]. 16.87\% [25].

Variation in the reported incidences of Yq microdeletion could be due to the difference in sample size variability and ethnicity. In our study, we used a multiplex PCR technique detecting 25 different STS in 5 multiplex PCR reactions for each patient. Using a higher sensitivity technique leads to increase in detection limit of $\mathrm{Y}$ microdeletions which explains the difference between our results and previous studies that reported a lower incidence of microdeletions due to using PCR technique detecting 6 STS [22], 10 STS[26]

Fourty seven percent of $\mathrm{Y}$ microdeletions in our study were detected in the $\mathrm{AZFb}+\mathrm{c}$, Thirty eight percent of microdeletions were identified in AZF-c patients, 9.5\% in AZF-a+b+c and only 4.8\% in AZF-b. Our data are showed that the highest incidence in Y microdeletions was in AZF-b+c; most of international reports revealed that, deletions of AZFc were at highest frequency Deletions in AZFc region was the most frequent 75\% [27], (48.1\%) [28], $(46.6 \%)$ [29].Variation in the reported frequencies could be related to the difference in ethnicity and sample size variability. 
In our study, among 114 azoospermic patients, 21/114 (18.4\%) had detectable Y chromosome microdeletions vs. only $3 / 36$ patients $(8.3 \%)$ in the oligospermic group. Supporting our results, previous reports revealed a comparable incidence of $16 \% 13.5 \%$, $12.8 \%, 10 \%$ and $6.4 \%$ in azoospermic patients and1.5\%, 8\%,8.8\%,2\% and $5.8 \%$ in oligospermic patients).[23, 31, 30,21, 29] respectively.

According to Asadi et al., 2017 [22] Concerning histopathological patterns; 3/21 patients (14.2\%) showed a hypospermatogenic pathology, 7/21 (33.3\%) showed C1 arrest while 7/21 (33.3\%) patient was sertoli cell only and 4/21 (19\%) showed mixed sertoli testicular pathology. It was previously stated that AZF deletions were associated with altered testicular histological characteristics which ranged from sertoli cell only to hypospermatogenesis. Within patients having Y microdeletion; 5/6 (83\%), 4/6 (67\%) and 2/6 (33\%) showed normal FSH, LH and testosterone, respectively. A previous report revealed a normal FSH, LH and testosterone levels in $36 \%$ of patients with Y microdeletions.

TESE extraction showed two successful trials while seventeen trails were negative. Both patients were NOA and had AZFc microdeletions which indicates that AZFc is a god prognosis for successful TESE outcomes.

\section{Conclusion:}

We can conclude that the frequency of $\mathrm{Y}$ chromosome microdeletions in our studied patients was similar to many ethnic reports. Detection of AZF microdeletions is necessary for proper genetic diagnosis in infertile males. AZFc can help informed decisions regarding positive TESE outcome.

\section{Conflict of interest:}

The authors declare no conflict of interest.

\section{References:}

[1] Agarwal A, Mulgund A, Hamada A, Chyatte MR. A unique view on male infertility around the globe. Reproductive Biology and Endocrinology. 2015 Apr 26; 13.

[2] Jodar M, Soler-Ventura A, Oliva R. Semen proteomics and male infertility. Journal of Proteomics. 2017Jun; 162:125-34.

[3] Anwar S, Anwar A, Karachi S, Pakistan. Infertility: A Review on Causes, Treatment and Management .2016 d-management.pdf

[4] Sharma A. Male Infertility; Evidences, Risk Factors, Causes, Diagnosis and Management in Human. Annals of Clinical and Laboratory Research. 2017.

[5] Colaco S, Modi D. Genetics of the human Y chromosome and its association with male infertility. Reproductive Biology and Endocrinology. 2018 Feb 17. 
[6] Yu X-W, Wei Z-T, Jiang Y-T, Zhang S-L. Y chromosome azoospermia factor region microdeletions and transmission characteristics in azoospermic and severe oligozoospermic patients. International journal of clinical and experimental medicine .2015; 8(9):14634-46.

[7] Alechine E, Corach D. High-Throughput Screening for Spermatogenesis Candidate Genes in the AZFc Region of the Y Chromosome by Multiplex Real Time PCR Followed by High Resolution Melting Analysis. PLOS ONE. 2014 May 14; 9(5):e97227.

[8] Ferlin A, Moro E, Rossi A, Dallapiccola B, Foresta C. The human Y chromosome's azoospermia factor $\mathrm{b}(\mathrm{AZFb})$ region: sequence, structure, and deletion analysis in infertile men. J Med Genet. 2003 Jan 1;40(1):18-24.

[9] Bhowmick BK, Satta Y, Takahata N. The origin and evolution of human ampliconic gene families and ampliconic structure. Genome Res. 2007 Apr; 17(4):441-50.

[10] Behulova R, Varga I, Strhakova L, Bozikova A, Gabrikova D, Boronova I, et al. Incidence of microdeletions in the AZF region of the $\mathrm{Y}$ chromosome in Slovak patients with azoospermia. Biomedical papers of the Medical Faculty of the University Palacky, Olomouc, Czechoslovakia. 2011;155(1):33-8.

[11] Tiepolo L, Zuffardi O. Localization of factors controlling spermatogenesis in the nonfluorescent portion of the human Y chromosome long arm. Hum Genet. 1976 Oct 28;34(2):119-24.

[12]Calogero AE, Garofalo MR, Barone N, Longo GA, De Palma A,Fichera M, et al. Spontaneous transmission from a father to his son of a $\mathrm{Y}$ chromosome microdeletion involving the deleted in azoospermia (DAZ) gene. J Endocrinol Invest.2002 Aug;25(7):631-4.

[13] Silber SJ. The Y chromosome in the era of intracytoplasmic sperm injection: a personal review. Fertil Steril. 2011 Jun 30;95(8):2439-2448-5.

[14] Edition F. Examination and processing of human semen. World Health Ed F 10 [Internet]. 2010 [cited 2017 Jun 19];286.

[15]Abdullah L, Bondagji N. Histopathological patterns of testicular biopsy in male infertility: A retrospective study from a tertiary care center in the western part of Saudi Arabia. Urol Ann. 2011; 3(1):19-2

[16]Schoch C, Schnittger S, Bursch S, Gerstner D, Hochhaus A, Berger U, et al. Comparison of chromosome banding analysis, interphase- and hypermetaphase-FISH, qualitative and quantitative PCR for diagnosis and for follow-up in chronic myeloid leukemia: a study on 350 cases. Leukemia. 2002 Jan;16(1):53-9.

[17] Omrani MD, Samadzadae S, Bagheri M, Attar K. Y chromosome microdeletions in idiopathic infertile men from West Azarbaijan. Urol J. 2006;3(1):38-43.

[18] Malekasgar AM, Mombaini H. Screening of "Y" chromosome microdeletions in Iranian infertile males. J Hum Reprod Sci. 2008 Jan;1(1):2-9. 
[19] Mirfakhraie R, Mirzajani F, Kalantar SM, Montazeri M, Salsabili N, Pourmand GR, et al. High prevalence of $\mathrm{AZFb}$ microdeletion in Iranian patients with idiopathic non-obstructive azoospermia. Indian J Med Res. 2010 Sep; 132:265-70.

[20] Zaimy MA, Kalantar SM, Sheikhha MH, Jahaninejad T, Pashaiefar H, Ghasemzadeh J, et al. The frequency of Yq microdeletion in azoospermic and oligospermic Iranian infertile men. Iran J Reprod Med. 2013 Jun;11(6):453-8.

[21] ElNahass YH, Ahwany A, Mekky Y, Fayez MS, Mohasseb EM, ElRefaey FA.(2017): YChromosome Microdeletions and their association with male factor infertility in Egyptian Patients. Life Science Journal ;14(6)

[22] Asadi F, Sadighi Gilani MA, Ghaheri A, Roodgar Saffari J, Zamanian M. The Prevalence of Y Chromosome Microdeletions in Iranian Infertile Men with Azoospermia and Severe Oligospermia. Cell J Yakhteh. 2017;19(1):27-33.

[23] Oliva R, Margarit E, Ballescá J-L, Carrió A, Sánchez A, Milà M, et al. Prevalence of Y chromosome microdeletions in oligospermic and azoospermic candidates for intracytoplasmic sperm injection. Fertility and Sterility. 1998 Sep;70(3):506-10.

[24] Mahanta R, Gogoi A, Roy S, Bhattacharyya IK, Sharma P. Prevalence of azoospermia factor (AZF) deletions in idiopathic infertile males in north-east India. Int $\mathbf{J}$ Hum Genet. 2011;11(2):99-104.

[25] Zhang Y-S, Dai R-L, Wang R-X, Zhang Z-H, Fadlalla E, Liu R-Z. Azoospermia factor microdeletions: occurrence in infertile men with azoospermia and severe oligozoospermia from China. Andrologia. 2014 Jun;46(5):535-40.

[26] Tabassum Siddiqui R, Mujtaba N, Naz M. The prevalence of Y chromosome microdeletions in Pakistani infertile men. Iranian journal of reproductive medicine 2013];11(8):619-24.

[27] Elfateh F, Rulin D, Xin Y, Linlin L, Haibo Z, Liu R-Z. Prevalence and patterns of Y chromosome microdeletion in infertile men with azoospermia and oligzoospermia in Northeast China. Iranian journal of reproductive medicine. 2014;12(6):383-8.

[28]Akinsal EC, Baydilli N, Dundar M, Ekmekcioglu O. The frequencies of Y chromosome microdeletions in infertile males. Türk Üroloji Dergisi/Turkish Journal of Urology. 2018; 44(5):389-92.

[29] Sen S, Pasi AR, Dada R, Shamsi MB, Modi D. Y chromosome microdeletions in infertile men: prevalence, phenotypes and screening markers for the Indian population. Journal of Assisted Reproduction and Genetics. 2013;30(3):413-22.

[30] Ambulkar PS, Sigh R, Reddy M, Varma PS, Gupta DO, Shende MR, et al. Genetic Risk of Azoospermia Factor (AZF) Microdeletions in Idiopathic Cases of Azoospermia and Oligozoospermia in Central Indian Population. J Clin Diagn Res JCDR. 2014 Mar;8(3):8891. 
[31]Balkan M, Tekes S, Gedik A. Cytogenetic and Y chromosome microdeletion screening studies in infertile males with Oligozoospermia and Azoospermia in Southeast Turkey. J Assist Reprod Genet. 2008 Nov;25(11-12):559-65. 


\section{الملخص باللغةة العريية}

دراسة مسحية لتعيين الحُذف الجينية الاقيقة للكروموسوم الصبغي الجنسي في الرجال المصريين العقماء بأستخدام تفاعل البلمرة المتسلسل المتعدد

الخلفية: من الأسباب الرئيسية الوراثية في العقم عند الرجال هي الخلل الكروموسومي والحُذف الجينية

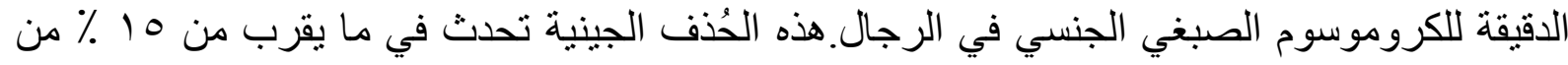

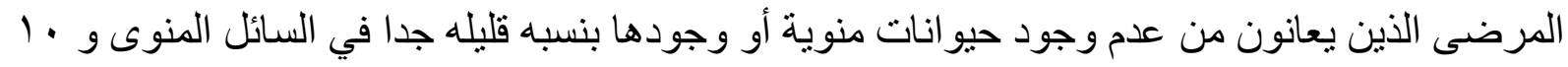

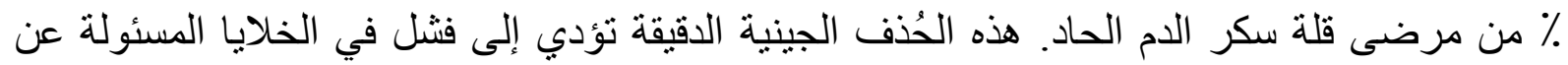

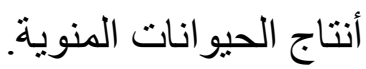

طرق البحث: أجري البحث علي •10 من الرجال المصابين بالعقم وتقسيمه ألي مجمو عتين أساسيتين

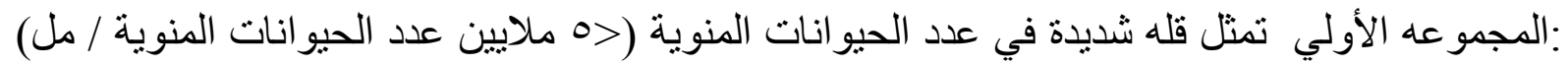

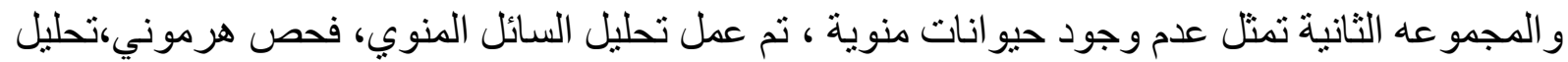

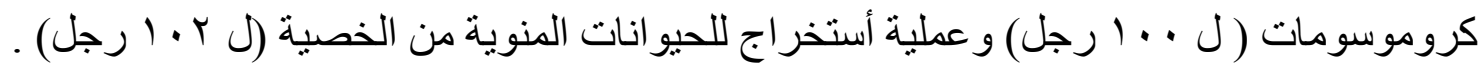

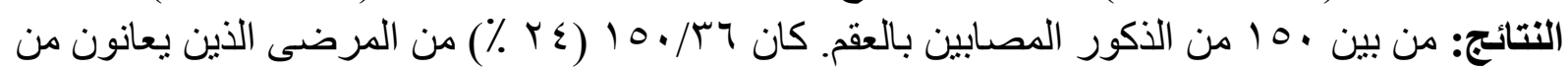

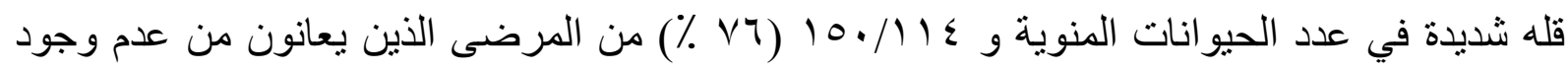
حيوانات منوية. بالنسبة للفحص الهرموني: لم تكن هنالك فروق ذات دلاتلة دالة إحصائية في المستويات

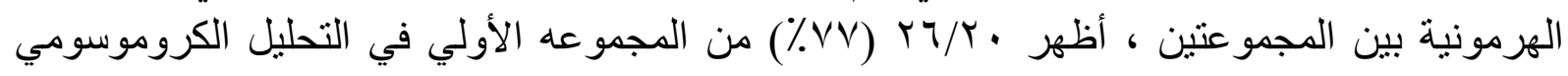

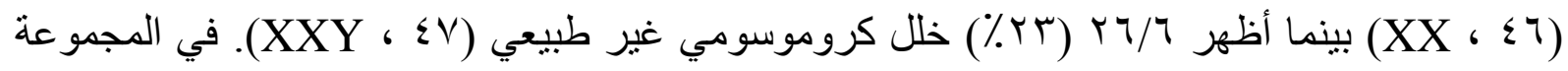

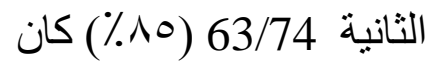

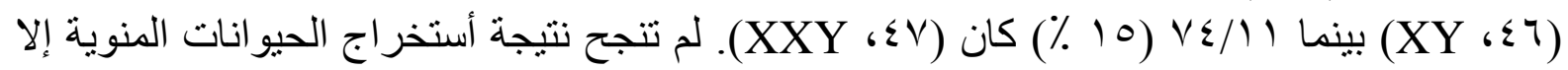

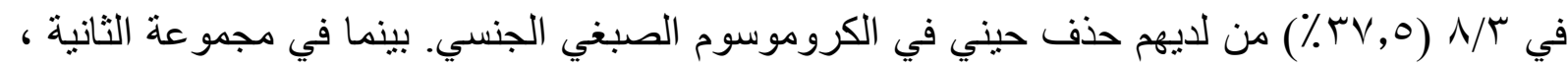

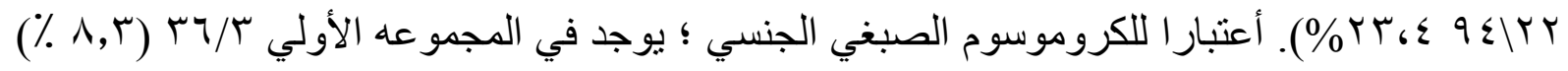

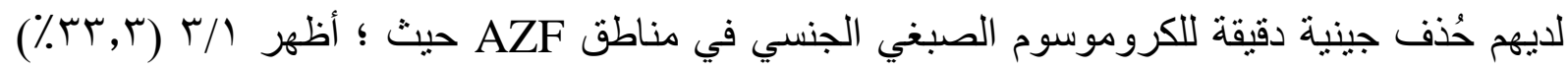

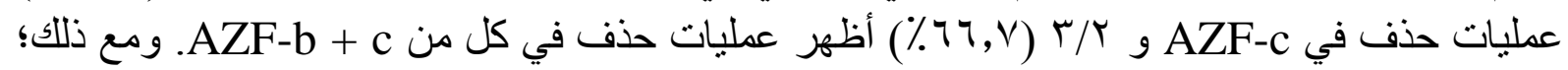

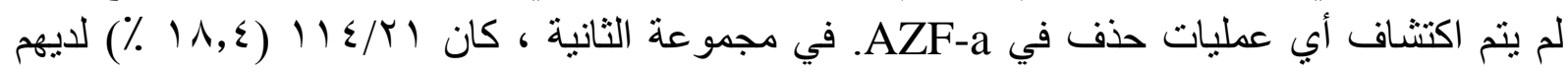

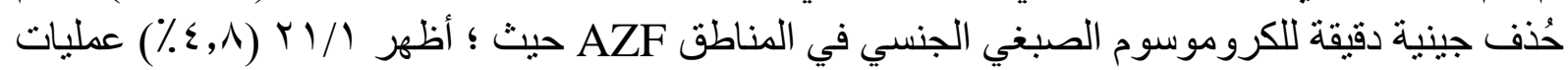

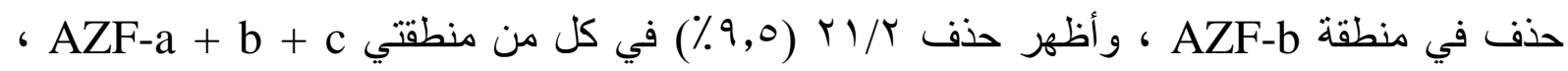

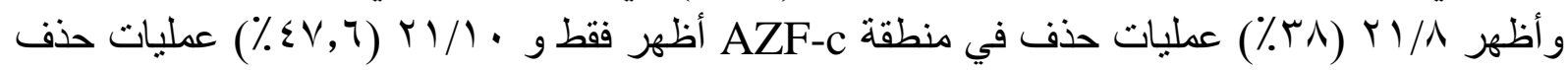

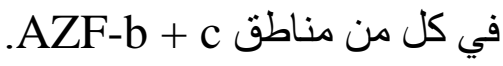
الخلاصه: كان معدل حدوث الحُذف الجينية الدقيقة للكروموسوم الصبغي الجنسي في الرجال في

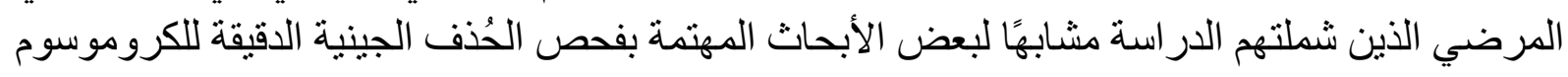
الصبغي الجنسي في الرجال. [19] [25] الكثف عن الحُذف الجينية الدقيقة للكروموسوم الصبغي الجنسي ضروري للتنشيص الور اثي السليم في الذكور المصابين بالعق. وجود حُذف جينية دقيقة في منطقة AZFc يمكن أن تساعد في اتخاذ قرارات مستنيرة بثأن نتائج إيجابية في عملية أستخر اج الحيو انات المنوية من الخصية. 